\title{
Tirzepatide: A Useful Addition for Treatment of Type 2 Diabetes and Obesity
}

\section{Nasser Mikhail}

Endocrinology division, Department of Medicine, OliveView-UCLA Medical Center, David-Geffen UCLA Medical School, CA, USA.

Corresponding Author: Nasser Mikhail, MD, MSc, Chief Endocrinology Division, Olive View-UCLA Medical Center, Sylmar, CA, USA.

Received date: August 08, 2021; Accepted date: August 25, 2021; Published date: September 09, 2021

Citation: Mikhail N. (2021) Tirzepatide: A Useful Addition for Treatment of Type 2 Diabetes and Obesity J. Gastroenterology Pancreatology and Hepatobilary Disorders. 5(5) DOI: 10.31579/2641-5194/044

Copyright: (c) 2021, Nasser Mikhail, This is an open access article distributed under the Creative Commons Attribution License, which permits unrestricted use, distribution, and reproduction in any medium, provided the original work is properly cited.

\begin{abstract}
Background: Tirzepatide is a dual receptor agonist of the 2 incretin hormones: glucose-dependent insulinotropic polypeptide (GIP) and glucagon-like peptide-1 (GLP-1) which is currently under development.

Objective: To clarify the potential role of tirzepatide for treatment of type 2 diabetes and obesity.

Methods: Pubmed search until August 5th, 2021. Search terms were GLP-1, GIP, incretins, tirzepatide, efficacy, safety. Clinical trials and pertinent reviews were included.

Results: In one phase 3 clinical trial, mean reduction of glycated hemoglobin (HbA1c) with tirzepatide (5-15 mg/week) was -1.87 to $-2.07 \%$ versus $+0.04 \%$ with placebo after 40 weeks. In another phase 3 randomized trial, tirzepatide decreased $\mathrm{HbA} 1 \mathrm{c}$ levels by $-2.01 \%$ to $-2.3 \%$ versus $-1.86 \%$ with semaglutide $(1.0 \mathrm{mg} /$ week $)(\mathrm{P}<0.0001)$. In the previous 2 studies, tirzepatide use was associated with dose-related mean weight loss at 40 weeks of $-7.0 \mathrm{~kg} 0-9.5 \mathrm{~kg}$ vs $-0.7 \mathrm{~kg}$ with placebo $(\mathrm{P}<0.0001)$ and $-7.6 \mathrm{~kg}$ to $-11.2 \mathrm{~kg}$ vs $-5.7 \mathrm{~kg}$ with semaglutide $((\mathrm{P}<0.001)$. Tirzepatide had generally favorable effects on lipid profile, particularly on lowering serum triglyceride levels: $-19 \%$ to $-24.8 \%$ mean reduction compared with $-11.5 \%$ with semaglutide. Gastrointestinal (GI) adverse effects were the commonest reported symptoms associated with tirzepatide use and occurred more frequently than with placebo and semaglutide. Hypoglycemia was reported more coomonly with high-dose terzipatide (1.7\%) compared with semaglutide $(0.4 \%)$. Drug discontinuation rates due to adverse effects were $6-8.5 \%$ with tirzepatide vs $4.1 \%$ with semaglutide.
\end{abstract}

Conclusions: Available data from 2 short-term randomized trials suggest that tirzepatide may be more effective than placebo and the GLP-1 agonist semaglutide in reducing HbAlc levels and body weight. Pattern of safety profile of tirzepatide is similar to that of GLP-1 agonists, but frequency of GI adverse effects and hypoglycemia is more common with tirzepatide.

Key words: tirzepatide; incretins ; semaglutide ; GLP-1; GIP; efficacy; safety; weight loss

\section{Introduction}

Tirzepatide (LY3298176) is a dual GIP/GLP-1 receptor agonist [1]. Its 39 amino acid sequence is mainly based on that of native GIP which consists of 42 amino acids [1]. Tirzepatide is attached to a 20-carbon fatty diacid moiety which binds to albumin. Albumin binding prolongs its half-life to approximately 5 days allowing once a week subcutaneous administration [2]. Tirzepatide has similar GIP receptor binding affinity to native GIP. Yet, it has 5 times lower affinity to GLP-1 receptor compared to native GLP-1 [2]. Thus, this drug was described as an imbalanced dual agonist in favor of GIP receptor over GLP-1 receptor activity [3]. Tirzepatide is currently under investigation in a series of phase 3 clinical trials (called SURPASS-1 through 6) [4]. Primary end point in these trials is the change in $\mathrm{HbA} 1 \mathrm{c}$ levels from baseline to the end of treatment with tirzepatide versus comparator. SURPASS-1 and 2 trials were recently published [5, 6]. These studies are summarized in table 1 and represent the focus of this review.

\section{Mechanism of action of tirzepatide}

Being a dual agonist of receptors of GLP-1 and GIP, tirzepatide would be expected to exert actions similar to those occurring subsequent to activation of these 2 receptors. However, the mechanism of action of tirzepatide does not reflect several agonistic effects of GIP. For instance, while both GLP-1 and GIP stimulate insulin secretion in healthy individuals, efficacy of GIP as anti-hyperglycemic agent in patients with 
type 2 diabetes may be severely compromised [7, 8]. Paradoxically, in some studies, GIP may even worsen post prandial hyperglycemia [9]. The latter effect may be at least in part due the finding that GIP, contrary to GLP-1, acutely increased glucagon plasma levels [7, 10]. Moreover, when administered to patients with type 2 diabetes receiving long-term treatment with the GLP-1 receptor agonist liraglutide, GIP did not acutely decrease energy intake and appetite nor increased energy expenditure, but increased plasma glucose and glucagon levels [10]. In addition, data regarding effects of GIP on weight loss are conflicting. Thus, GIP may enhance deposition of fat in adipose tissues and was therefore considered the "obesity hormone" [1, 11]. Finally, while GLP-1 delays gastric emptying, GIP does not exert such effect [7, 12]. To explain this paradox, some authors hypothesize that chronic activation of receptors of both GLP-1 and GIP by tirzepatide could result in desensitization of GIP receptors, which ultimately results in creating GIP antagonism [1].

Nevertheless, available clinical data have shown that terzipatide may improve insulin sensitivity. This was shown by the decrease in insulin resistance by tirzepatide vs placebo in the homeostatic model assessment of insulin resistance (HOMA-IR) in SURPASS-1 trial [5]. This amelioration in insulin sensitivity is caused in small part by weight loss. Thus, in one study, weight reduction explained $13 \%$ and $21 \%$ of improvement HOMA-IR with tirzepatide doses of 10 and $15 \mathrm{mg}$, respectively [13]. On the other hand, Fonesca et al [14] have shown that reduction in insulin resistance by semaglutide is primarily mediated by weight loss in subjects with type 2 diabetes.

\section{Anti-hyperglycemic efficacy of tirzepatide}

\section{Tirzepatide versus placebo}

SURPASS-1 trial is a double-blind randomized trial that included 478 overweight/obese patients (mean weight $85.9 \mathrm{~kg}$ ) with type 2 diabetes inadequately controlled on diet and exercise alone (mean HbA1c 7.9\%) (Table 1) [5]. Participants were randomly assigned to once weekly tirzepatide $5 \mathrm{mg}, 10 \mathrm{mg}, 15 \mathrm{mg}$ or placebo [5]. After 40 weeks, mean HbA1c levels decreased from baseline by $-1.87,-1.89$, and 2.07 percentage points with tirzepatide $5 \mathrm{mg}, 10 \mathrm{mg}$ and $15 \mathrm{mg}$, respectively vs a slight increase of +0.04 percentage points with placebo (all $\mathrm{P}<0.0001$ versus placebo) (table 1) [5].

\section{Tirzepatide versus semaglutide}

In SURPASS-2 trial, patients with type 2 diabetes on metformin ( $\mathrm{n}=1,878$, mean weight $93.7 \mathrm{~kg}$, mean $\mathrm{HbA1c} 8.2 \%$ ) were randomized to receive once weekly tirzepatide $5 \mathrm{mg}, 10 \mathrm{mg}, 15 \mathrm{mg}$ or the GLP-1 receptor agonist semaglutide $1 \mathrm{mg}$ once weekly in an open-label fashion (table 1) [6]. At 40 weeks, mean reductions from baseline in HbA1c levels with tirzepatide $5 \mathrm{mg}, 10 \mathrm{mg}$, and $15 \mathrm{mg}$ were $-2.01,-2.24$ and -2.30 percentage points, respectively, as compared with -1.86 percentage points with semaglutide [6]. In fact, all terzipatide doses were statistically superior to semaglutide. In all treatment groups, HbA1c levels reached a trough after 24 weeks followed by a plateau [6].

\section{Effects of tirzepatide effect on body weight}

Tirzepatide exerts a potent dose-related effect on weight loss that was far superior to that caused by semaglutide $(-11.2 \mathrm{~kg}$ vs $-5.7 \mathrm{~kg})$ (table 1) [5.6]. Weight loss was observed as early as 4 weeks $[5,6]$. Contrary to their effects on HbA1c levels that plateaued after 24 weeks, weight reduction associated with tirzepatide and semaglutide use continued until the end of intervention at 40 weeks [5, 6]. Mechanisms of weight loss associated with terzipatide use are not fully understood. Surprisingly, in SURPASS2 , decreased appetite was reported by only $0.4 \%$ in patients receiving tirzepatide and none of patients receiving semaglutide [6]. In SURPASS 1 , decreased appetite was reported by $4-8 \%$ randomized to semaglutide versus $1 \%$ with placebo [5]. One factor that may contribute to weight loss induced by tirzepatide is the relatively common occurrence of nausea and vomiting associated with its use (table 1). Comparison of the extent of weight loss between patients who reported GI adverse effects with tirzepatide versus those who did not may clarify this issue. Unfortunately, investigators in the SURPASS-1 and -2 trials did not report such important information $[5,6]$.

\section{Overview of SURPASS-1 and 2 trials of terzipatide}

\begin{tabular}{|c|c|c|}
\hline & SURPASS-1 [5] & SURPASS-2 [6] \\
\hline Design & $\begin{array}{l}\text { Randomized, double-blind, placebo- } \\
\text { controlled multinational, } 40 \text { weeks, } \mathrm{n}=478\end{array}$ & $\begin{array}{l}\text { Randomized, open-label, multinational, } 40 \text { weeks, } \\
\mathrm{n}=1,879\end{array}$ \\
\hline Patients' characteristics & $\begin{array}{l}\text { Age } 54.1 \text { years, } 48 \% \text { women, BMI } 31.9 \\
\mathrm{~kg} / \mathrm{m}^{2}\end{array}$ & Age 56.6 years, $53 \%$ women, BMI $34.2 \mathrm{~kg} / \mathrm{m}^{2}$ \\
\hline Diabetes background drug therapy & None & Metformin \\
\hline Baseline mean $\mathrm{Hg} \mathrm{A} 1 \mathrm{c}$ & $7.9 \%$ & $8.28 \%$ \\
\hline Patient groups & $\begin{array}{l}\text { T5mg }(n=121), \text { T10mg }(n=121), \text { T15mg } \\
(n=121) P(n=115) .\end{array}$ & $\begin{array}{l}\text { T5mg }(n=470), \text { T10mg }(n=469), \text { T15mg }(n=470), S \\
(n=469)\end{array}$ \\
\hline $\begin{array}{l}\text { Changes in mean HbA1c vs baseline (primary } \\
\text { endpoint) }\end{array}$ & $\begin{array}{l}\text { T5mg }-1.87 \%, \text { T10mg }-1.89 \%, \text { T } 15 \mathrm{mg}- \\
2.07 \% \text { and } \mathrm{P}+0.04 \%(\mathrm{p}<0.0001 \mathrm{vs} \mathrm{P})\end{array}$ & $\begin{array}{l}\text { T5mg }-2.01 \%, \text { T10mg }-2.24 \%, \text { T15mg }-2.30 \%, S- \\
1.86 \text { (p<0.001 vs } S) .\end{array}$ \\
\hline Changes in weight (kg) & $\begin{array}{l}\text { T5 mg -7.0, T10 mg -7.8, T15mg -9.5, P - } \\
0.7 \text { (p<0.0001 vs P) }\end{array}$ & $\begin{array}{l}\text { T5mg -7.6kg, T10mg }-9.3 \mathrm{~kg}, \mathrm{~T} 15 \mathrm{mg}-11.2 \mathrm{~kg}, \mathrm{~S}- \\
5.7 \mathrm{~kg}(\mathrm{p}<0.001 \mathrm{vs} \mathrm{S}) .\end{array}$ \\
\hline Drug discontinuation due to adverse effects & T5mg 3\%, T10mg 5\%, T15mg 7\%, P 3\% & T5mg 6.0\%, T10mg 8.5\%, T15mg 8.5\%, S 4.1\% \\
\hline Nausea & $\begin{array}{l}\text { T5mg } 12 \%, \text { T10 mg 13\%, T15 mg 18\%, P } \\
6 \%\end{array}$ & $\begin{array}{l}\text { T5mg } 17.4 \% \text {, T10mg } 19.2 \% \text {, T15mg } 22.1 \%, \mathrm{~S} \\
17.9 \%\end{array}$ \\
\hline Diarrhea & $\begin{array}{l}\text { T5mg } 12 \%, \text { T10mg } 14 \% \text {, T15mg12\%, P } \\
8 \%\end{array}$ & $\begin{array}{l}\text { T5mg } 13.2 \% \text {, T10mg } 16.4 \% \text {, T15mg } 13.8 \% \text {, S } \\
11.5 \%\end{array}$ \\
\hline Vomiting & T5mg 3\%, T10mg 2\%, T15mg $6 \%$, P $2 \%$ & T5mg 5.7\%, T10mg 8.5\%, T15mg 9.8\%, S 8.3\% \\
\hline *Hypoglycemia & T5mg 0\%, T10 mg 0\%, T15 mg 0\%, P 1\% & T5mg 0.6\%, T10 mg 0.2\%, T15 mg 1.7\%, S $0.4 \%$ \\
\hline Injection site reactions & T5mg 3\%, T10mg 3\%, T15mg 2\%, P 0\% & T5mg $1.9 \%$, T10mg $2.8 \%$ T15 mg 4.5\%, S $0.2 \%$ \\
\hline
\end{tabular}




\author{
Abbreviations: \\ BMI: body mass index (mean), HbA1c: glycated hemoglobin \\ T: tirzepatide, S: semaglutide $1 \mathrm{mg}, \mathrm{P}$ : placebo. Previous medications were taken once weekly subcutaneously \\ Age and body mass index values are means. \\ Hypoglycemia: defined as blood glucose $<54 \mathrm{mg} / \mathrm{dl}$
}

\section{Effects of tirzepatide on lipid profile}

The main effect of tirzepatide on lipid panel is reduction of serum triglycerides $[5,6,15]$. In a post-hoc analysis of a phase 2 clinical trial, Wilson et al [15] showed that tirzepatide decreased plasma levels of apolipoprotein C III (apo III), the main regulator of triglyceride metabolism. In addition, terzipatide treatment increased serum mass of lipoprotein lipase, the enzyme responsible for clearance of circulating triglycerides [15]. Moreover, the decrease in apo CIII levels by tirzepatide emerged as the best predictor of changes in plasma triglycerides, whereas weight loss was a weak predictor for reduction in serum triglycerides [15]. In SURPASS-2, tirzepatide treatment was associated with dose-related significant reduction in serum triglycerides at 40 weeks vs baseline ranging from $-19.0 \%$ to $-24.8 \%$ compared with $-11.5 \%$ with semaglutide (statistical significance not reported) [6]. However, no significant differences were found between tirzepatide and semaglutide in total cholesterol and low-density lipoprotein cholesterol (LDL-C) levels [6]. Regarding high-density lipoprotein cholesterol (HDL-C) levels, there was $6.8-7.9 \%$ increase in the terzipatide groups at 40 weeks versus $4.4 \%$ increase with semaglutide (statistical significance was not mentioned). In the post-hoc analysis by Wilson et al [15], tirzepatide decreased small atherogenic LDL particles more than the GLP-1 agonist dulaglutide. Taken together, the above data suggest that tirzepatide has favorable effects on lipid profile.

\section{Safety of tirzepatide}

Overall, terzipatide was fairly tolerated. However, in phase 3 trials, the drug discontinuation rates due to adverse effects among patients randomized to tirzepatide (10-15 mg) were approximately double those recorded among subjects randomized to placebo (5-7\% vs 3\%) and semaglutide $(8.5 \%$ vs $4.1 \%)$ (Table 1$)[5,6]$.

\section{Gastrointestinal adverse effects}

The most common adverse effects of tirzepatide are related to the GI system, namely nausea, diarrhea and vomiting (table 1). They are doserelated, generally described as mild to moderate, and mainly occur in the first few weeks-months after starting therapy $[5,6]$. Starting tirzepatide in small doses of $2.5 \mathrm{mg} /$ week followed by slow up-titration (increase by 2.5 mg every 4 weeks) may be associated with decreased frequency of nausea compared with more rapid titration (starting by $5 \mathrm{mg} / \mathrm{week}$, then doubling the dose after 2 weeks, then $15 \mathrm{mg}$ after 4 weeks) [5,6,16]. When compared with semaglutide $1.0 \mathrm{mg}$, GI adverse effects were slightly more common with the highest dose of terzipatide $(15 \mathrm{mg}$ ) (table 1). Nausea and vomiting are attributed in part to delay in gastric emptying, which usually subsides after several weeks [17]. In fact, Urva et al [17] have shown that tirzepatide caused delay in gastric emptying that was similar in magnitude to the selective GLP-1 agonist dulaglutide. This finding suggests that delay in gastric emptying is mainly driven by the GLP-1 receptor component of terzipatide.

\section{Hypoglycemia}

The incidence of clinically significant hypoglycemia (defined as blood glucose levels $<54 \mathrm{mg} / \mathrm{dl}$ ) was low with tirzepatide use and similar to placebo (table 1) [5]. However, in SURPASS-2, the frequency of clinically significant hypoglycemia was approximately 4 times greater with tirzepatide $15 \mathrm{mg}$ when compared with semaglutide $(1.7 \%$ and $0.4 \%$, respectively) (table 1) [6]. Similarly, frequency of hypoglycemia defined as $\mathrm{BG}<70 \mathrm{mg} / \mathrm{dl}$ was numerically greater with tirzepatide $(7.5-9.8 \%) \mathrm{vs}$ dulaglutide $(3.7 \%)$, but no severe hypoglycemia was reported in any treatment group [16].

\section{Limitations of tirzepatide}

Despite the encouraging preliminary results reported with tirzepatide use, this drug has several limitations. First, data is limited to 2 phase 3 trials lasting only 40 weeks. Thus, it is not known whether reductions of hyperglycemia and weight are durable. Second, patients with chronic kidney disease (CKD) with estimated glomerular filtration rate (eGFR) below $45 \mathrm{ml} / \mathrm{min} / 1.73 \mathrm{~m}^{2}$ were excluded from these trials [5, 6]. One study by Urva et al [18] has shown that renal impairment including need for hemodialysis has no clinically relevant effects on pharmacokinetics of tirzepatide. However, the latter investigation was a single-dose study that used tirzepatide in a small dose of $5 \mathrm{mg}$ [18]. Third, patients with diabetic maculopathy and proliferative retinopathy and HbA1c levels > 10.5\% were also excluded. Fourth, long-term safety of tirzepatide, including its effects on cardiovascular and renal events, are not known.

\section{Conclusions and current directions}

No doubt, introduction of tirzepatide represents a welcome addition of a new class for potential treatment of type 2 diabetes and obesity. Available data that extend to 40 weeks suggest that this dual GLP-1 and GIP receptor agonist is clearly superior to selective GLP-1 agonists in terms of anti-hyperglycemic efficacy, weight loss, and reduction in levels of serum triglycerides. In general, tirzepatide has an acceptable safety profile. However, its use is associated with high frequency of GI adverse effects. Due to its superior efficacy in weight reduction, tirzepatide is currently being investigated as treatment for obesity in a randomized, double-blind trial called SURMOUNT-1 [4]. This trial is planning to recruit 2,400 subjects who do not have diabetes. The Federal Drug Administration (FDA) has recently approved in June 2021 high dose semaglutide (2.4 mg/week) (Wegovy ${ }^{\circledR}$ ) for treatment of obesity irrespective of presence of diabetes [19]. It would be interesting to compare tirzepatide and semaglutide ( $2.4 \mathrm{~g} /$ week) head to head as antiobesity agents. As weight loss is effective in treating non-alcoholic steatohepatitis (NASH), a phase 2 double-blind study (SYNERGY$\mathrm{NASH}$ ) is underway to evaluate tirzepatide for treatment of NASH [4].

Further data are needed to evaluate long-efficacy and safety of tirzepatide in various groups of patients with type 2 diabetes. For example, in poorly controlled type 2 diabetes patients (HbA1c >10\%), in patients with CKD and those receiving other concomitant diabetes therapy such as insulin or sodium-glucose co-transporters 2 (SGLT-2) inhibitors. The impact of tirzepatide on incidence of cardiovascular and renal events and mortality 
is certainly of crucial importance. This issue is currently under investigation in SURPASS-CVOT trial. The latter study is a large $(n=12,500)$ double-blind trial comparing tirzepatide with dulaglutide 1.5 $\mathrm{mg} /$ week and is expected to terminate in October 2024 [4].

\section{Conflicts of interest}

The author has no conflict of interest to declare.

\section{References}

1. Fukuda M. (2021) The Role of GIP Receptor in the CNS for the Pathogenesis of Obesity. Diabetes. Epub ahead of print.

2. Coskun T, Sloop KW, Loghin C, Alsina-Fernandez J, Urva S, et al. (2018) LY3298176, a novel dual GIP and GLP-1 receptor agonist for the treatment of type 2 diabetes mellitus: From discovery to clinical proof of concept. Mol Metab. 18:3-14.

3. Willard FS, Douros JD, Gabe MB, Showalter AD, Wainscott DB, et al. (2020) Tirzepatide is an imbalanced and biased dual GIP and GLP-1 receptor agonist. JCI Insight. 5(17):e140532

4. Min T, Bain SC. (2021) The Role of Tirzepatide, Dual GIP and GLP-1 Receptor Agonist, in the Management of Type 2 Diabetes: The SURPASS Clinical Trials. Diabetes Ther. 12(1):143-157.

5. Rosenstock J, Wysham C, Frías JP, Kaneko S, Lee CJ, et al. (2021) Efficacy and safety of a novel dual GIP and GLP-1 receptor agonist tirzepatide in patients with type 2 diabetes (SURPASS-1): a double-blind, randomised, phase 3 trial. Lancet. S0140-6736(21)01324-6.

6. Frías JP, Davies MJ, Rosenstock J, Pérez Manghi FC, Fernández Landó L, et al. (2021) SURPASS-2 Investigators. Tirzepatide versus Semaglutide Once Weekly in Patients with Type 2 Diabetes. $N$ Engl J Med. Epub ahead of print.

7. Seino Y, Fukushima M, Yabe D. (2010) GIP and GLP-1, the two incretin hormones: Similarities and differences. J Diabetes Investig. 1(1-2):8-23.

8. Elahi D, McAloon-Dyke M, Fukagawa NK, Meneilly GS, Sclater AL, et al. (1994) The insulinotropic actions of glucosedependent insulinotropic polypeptide (GIP) and glucagon-like peptide-1 (7-37) in normal and diabetic subjects. Regul Pept. 51(1):63-74.

9. Chia CW, Carlson OD, Kim W, Shin YK, Charles CP, Kim HS, Melvin DL, Egan JM. (2009) Exogenous glucose-dependent insulinotropic polypeptide worsens post prandial hyperglycemia in type 2 diabetes. Diabetes. 58(6):1342-9.
10. Bergmann NC, Gasbjerg LS, Heimbürger SM, Krogh LSL, Dela F, et al. (2020) No Acute Effects of Exogenous GlucoseDependent Insulinotropic Polypeptide on Energy Intake, Appetite, or Energy Expenditure When Added to Treatment with a Long-Acting Glucagon-Like Peptide 1 Receptor Agonist in Men with Type 2 Diabetes. Diabetes Care. 43(3):588-596.

11. Holst JJ, Rosenkilde MM. (2020) GIP as a Therapeutic Target in Diabetes and Obesity: Insight From Incretin Co-agonists. J Clin Endocrinol Metab. 105(8):e2710-6.

12. Meier JJ, Goetze O, Anstipp J, Hagemann D, Holst JJ, et al. (2004) Gastric inhibitory polypeptide does not inhibit gastric emptying in humans. Am J Physiol Endocrinol Metab. 286(4):E621-5.

13. Thomas MK, Nikooienejad A, Bray R, Cui X, Wilson J, et al. (2021) Dual GIP and GLP-1 Receptor Agonist Tirzepatide Improves Beta-cell Function and Insulin Sensitivity in Type 2 Diabetes. J Clin Endocrinol Metab. 106(2):388-396.

14. Fonseca VA, Capehorn MS, Garg SK, Jódar Gimeno E, Hansen $\mathrm{OH}$, et al. (2019) Reductions in insulin resistance are mediated primarily via weight loss in subjects with type 2 diabetes on semaglutide. J Clin Endocrinol Metab. 104 (9): 4078-4086.

15. Wilson JM, Nikooienejad A, Robins DA, Roell WC, Riesmeyer Js, et al. (2020) The dual glucose-dependent insulinotropic peptide and glucagon-like peptide-1 receptor agonist, tirzepatide, improves lipoprotein biomarkers associated with insulin resistance and cardiovascular risk in patients with type 2 diabetes. Diabetes Obes Metab. 22(12):2451-2459.

16. Frias JP, Nauck MA, Van J, Kutner ME, Cui X, et al. (2018) Efficacy and safety of LY3298176, a novel dual GIP and GLP-1 receptor agonist, in patients with type 2 diabetes: a randomised, placebo-controlled and active comparator-controlled phase 2 trial. Lancet. 392(10160):2180-2193.

17. Urva S, Coskun T, Loghin C, Cui X, Beebe E, et al. (2020) The novel dual glucose-dependent insulinotropic polypeptide and glucagon-like peptide-1 (GLP-1) receptor agonist tirzepatide transiently delays gastric emptying similarly to selective longacting GLP-1 receptor agonists. Diabetes Obes Metab. 22(10):1886-1891.

18. Urva S, Quinlan T, Landry J, Martin J, Loghin C. (2021) Effects of Renal Impairment on the Pharmacokinetics of the Dual GIP and GLP-1 Receptor Agonist Tirzepatide. Clin Pharmacokinet. Epub ahead of print.

19. Wegov (Semaglutide). (2021) Prescribing information. Novo Nordisk Inc., Plainsboro, NJ, June.

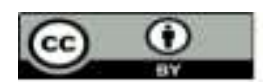

This work is licensed under Creative Commons Attribution 4.0 License
Ready to submit your research? Choose Auctores and benefit from:

$>$ fast, convenient online submission

$>$ rigorous peer review by experienced research in your field

$>$ rapid publication on acceptance

$>$ authors retain copyrights

$>$ unique DOI for all articles

$>$ immediate, unrestricted online access

At Auctores, research is always in progress.

Learn more auctoresonline.org/journals/gastroenterology-pancreatologyand-hepatobilary-disorders 